\title{
Near band gap photoreflectance studies in CdTe, CdTe:V and CdTe:Ge crystals
}

\author{
U. Pala , J.L. Herrera Pérez ${ }^{\mathrm{a}}$,J. Piqueras ${ }^{\mathrm{b}}$, E. Dieguéz ${ }^{\mathrm{c}}$ \\ ${ }^{a}$ Instituto de Física, Universidad Autónoma de Puebla, Apdo. Postal J 48, Puebla, Pue 72570, México

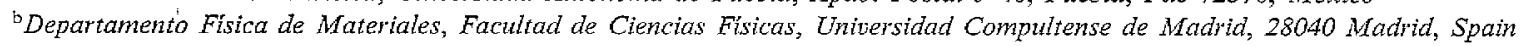 \\ 'Departamento de Física de Materiales, Universidad Autónoma, C-IV, 28049 Cantoblanco-Madrid, Spain
}

\begin{abstract}
Abstruct
The contactless modulation spectroscopy technique of photoreflectance (PR) has been used to study the near band edge transitions in CdTe, CdTe:V and CdTe:Ge bulk crystals in the range of 14 and $400 \mathrm{~K}$ for the first time. The lineshape of the PR spectra for the crystals is seen to follow the third derivative functional form (TDFF) of electroreflectance (ER) in the low field limit. Using the line shape analysis of the spectra at different temperatures, the variation of band gap $\left(E_{0}\right)$, phase factor $(\theta)$ and energy broadening parameter $(\Gamma)$ with temperature are studied. The temperature variation of band gap for these crystals is seen to follow the Varshni relation with coefficient values $\alpha=4.357 \times 10^{-4} \mathrm{eV} \mathrm{K}^{-1}, \beta=183.4 \mathrm{~K}$ for undoped, $\alpha=4.635 \times 10^{-4} \mathrm{eV}$ $\mathrm{K}^{-1}, \beta=184.5 \mathrm{~K}$ for vanadium-doped and $\alpha=4.508 \times 10^{-4} \mathrm{eV} \mathrm{K}-1, \beta=230.5 \mathrm{~K}$ for Ge doped crystals. The Varshni relation is found to be valid for the whole range of temperature studied for undoped and Ge-doped crystals, where as for V-doped crystals, Varshni relation is valid upto about $250 \mathrm{~K}$. Effects of vanadium and germanium doping on the energy broadening parameter in CdTe are discussed.
\end{abstract}

Keywords: Photoreflectance; Cadmium telluride

\section{Introduction}

Photoreflectance (PR) spectroscopy has been shown to be useful nondestructive and contactless technique for the characterization of semiconductors $[1-3]$ and semiconductor microstructures [4,5]. In the PR, the electric field in the materials is modulated by the photoinjection of electron-hole pairs by a secondary (pump) beam chopped at certain frequency. It has been demonstrated that PR is indeed a form of electroreflectance (ER) $[1,6]$ producing sharp third-derivative like spectra (low field regime) or Franz-Keldysh oscillations for large modulation or large built-in electric field in the bulk crystals or thin films.

The interest in detailed features of CdTe crystals arises from the extensive use of this compound in the fabrication of nuclear detectors, epitaxy substrates for $\mathrm{HgCdTe}$ and many other electro-optic devices. The prospect of their important practical usage imposes several physical requirements for choice of the crystals for varied applications. In particular, a high room temperature resistivity and a good photoconductivity on infrared irradiation are important requirements. The $\mathrm{CdTe}$ crystals are doped very often by vanadium and germanium impurities for achieving some of the desired properties $[7,8]$. These dopings compensate deep donor levels and thereby providing the semi-insulating $\mathrm{CdTe}$ crystals with improved characteristics in beam coupling experiments [7,9]. In addition, these samples exhibit good photorefractive properties [8] and also show fairly good nuclear detection resolution [10].

In the present paper we report the PR spectroscopic studies of CdTe, CdTe:V and CdTe:Ge crystals in the near band gap region between 14 and $400 \mathrm{~K}$. To our knowledge, there is no systematic study of PR spectra and their line shape analysis in CdTe:V and CdTe:Ge crystals in this temperature range. From the lineshape analysis of the PR spectra recorded at different temperatures, the variation of band gap, the amplitude, phase, and energy broadening parameter with temperature are studied for undoped, as well as for vanadium doped and germanium doped CdTe crystals. It is shown that $\Gamma$ value in C.CTe increases as a result of doping with different impurities. 


\section{Experimental}

The CdTe, CdTe: $\mathrm{V}$ and CdTe:Ge crystals used in the present study were grown by the vertical Bridgman technique with displacement of the furnace, using $\mathrm{Cd}$ and $\mathrm{Te}(6 \mathrm{~N} \mathrm{Caminco})$ as the starting materials. For the $\mathrm{V}$-doped and Ge-doped crystal, high purity $\mathrm{V}$ metal and $\mathrm{Ge}$ were added to the melt in a concentration of $7 \times 10^{19} \mathrm{~cm}^{-3}$ and $10^{19} \mathrm{~cm}^{-3}$, respectively. The wafers were prepared by mechanical polishing with alumina powder, followed by a chemo-mechanical polishing with $2 \%$ bromine-methanol solution and then rinsed in methanol. The resistivity of CdTe:V and CdTe:Ge crystals was $10^{8}-10^{9} \Omega \mathrm{cm}$ at room temperature.

For the PR studies, immediately after polishing, the samples were mounted on the cold finger of an evacuated cryostat (Air Products) in which the sample temperature could be varied from 14 to $400 \mathrm{~K}$. The experimental geometry of the PR setup is similar to that used by Shay [6]. The source of the probe beam was a tungsten-halogen $(150 \mathrm{~W})$ lamp. A Carl-Zeiss (Jena SPM-2) $0.30 \mathrm{~m}$ monochromator produced the monochromatic radiation focused on the crystal surface with a typical spot size of $6 \mathrm{~mm} \times 2 \mathrm{~mm}$. The pump beam was a $6328 \AA$ line of a $\mathrm{He}-\mathrm{Ne}$ laser with a power density of about $300 \mu \mathrm{W} \mathrm{cm}-2$ and chopped at $205 \mathrm{~Hz}$. The reflected light was focused onto a UDT $\mathrm{p}-\mathrm{i}-\mathrm{n}$ silicon photodiode operated in its photoconductive mode. A corning glass filter was placed in front of the detector to prevent the scattered laser light from reaching the detector. Both the $\mathrm{DC}$ and $\mathrm{AC}$ voltage levels of the detector were detected by a synchronized lock-in amplifier coupled with a current sensitive preamplifier (Princeton Applied Research, Model 184). These signals were monitored by a computer with which the $P R$ signal ratio $\Delta R / R$ was obtained at each wavelength, The computer also controlled the wavelength scan of the monochromator via a stepping motor. Signal averaging technique was employed at each wavelength to reduce the signal-to-noise ratio.

\section{Results and discussions}

The typical PR spectra for undoped CdTe crystal, recorded at different temperatures are shown in Fig. 1. The PR spectra obtained for the samples were analysed using the theory developed by Aspnes [11] for low field electroreflectance using the expression:

$\Delta R / R=\operatorname{Re}\left[C \mathrm{e}^{i \theta}\left(E-E_{0}+i \Gamma\right)^{-n}\right]$

where $E$ is the energy of the incident light, $E_{0}$ is the interband transition energy, $\Gamma$ is an energy broadening parameter, $C$ and $\theta$ are amplitude and phase factors which are only weakly dependent on $E$, and the exponent $n$ is the dimensionality of the critical point in- volved. The broadening energy $\Gamma$, is used as a measure of crystal quality since its magnitude is primarily determined by lattice defects such as disorder, vacancies, impurities, etc. [12]. For the present analysis, $n=2.5$ is chosen which corresponds to a three dimensional critical point.

In the temperature variation of PR spectra of undoped CdTe crystal a conventional decrease of $P R$ signal upto $160 \mathrm{~K}$ is observed which arises due to the reduction of built-in electric field with temperature. Beyond this temperature, the PR signal increased along with a change of phase. The increase of PR signal with the increase of temperature implies that the band. edge signal for such semi-insulating crystals comes from inherent traps, impurities or possible bound excitons. Similar observations have been made by Sydor et al. [13] for their semi-insulating GaAs substrates. The modulation mechanism responsible for such $P R$ arises either due to a thermal emptying of the traps, or thermal dissociation and subsequent momentary refilling of the traps by laser-injected carriers. In case of CdTe:V and CdTe:Ge crystals, the PR signal increased with the temperature throughout the investigated range of temperature.

In the TDFF fitting of PR spectra of undoped and vanadium doped crystals recorded at low temperatures, the high energy side of the spectra did not fit well with the corresponding experimental line shape. Two dips are observed in the low temperature PR spectra. The occurrence of an additional dip at the high energy side gives rise to a misfit between experimental and theoretical curves.

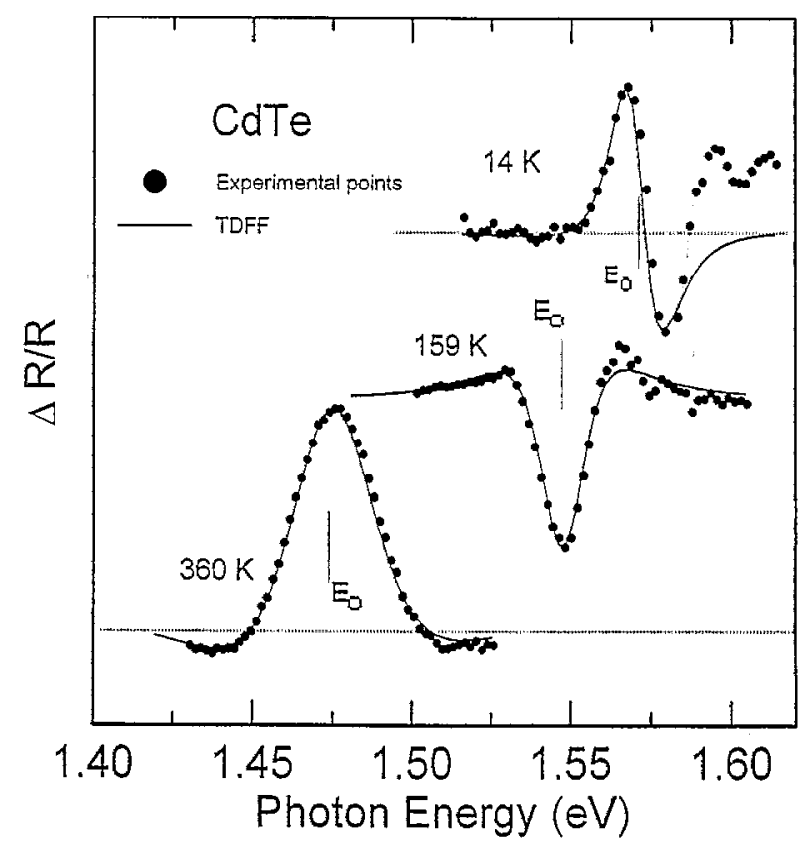

Fig. 1. Typical PR spectra of undoped CdTe crystal recorded at 14 , 159 and $360 \mathrm{~K}$. The vertical bars show the $E_{0}$ positions as evaluated from the lineshape analysis. 

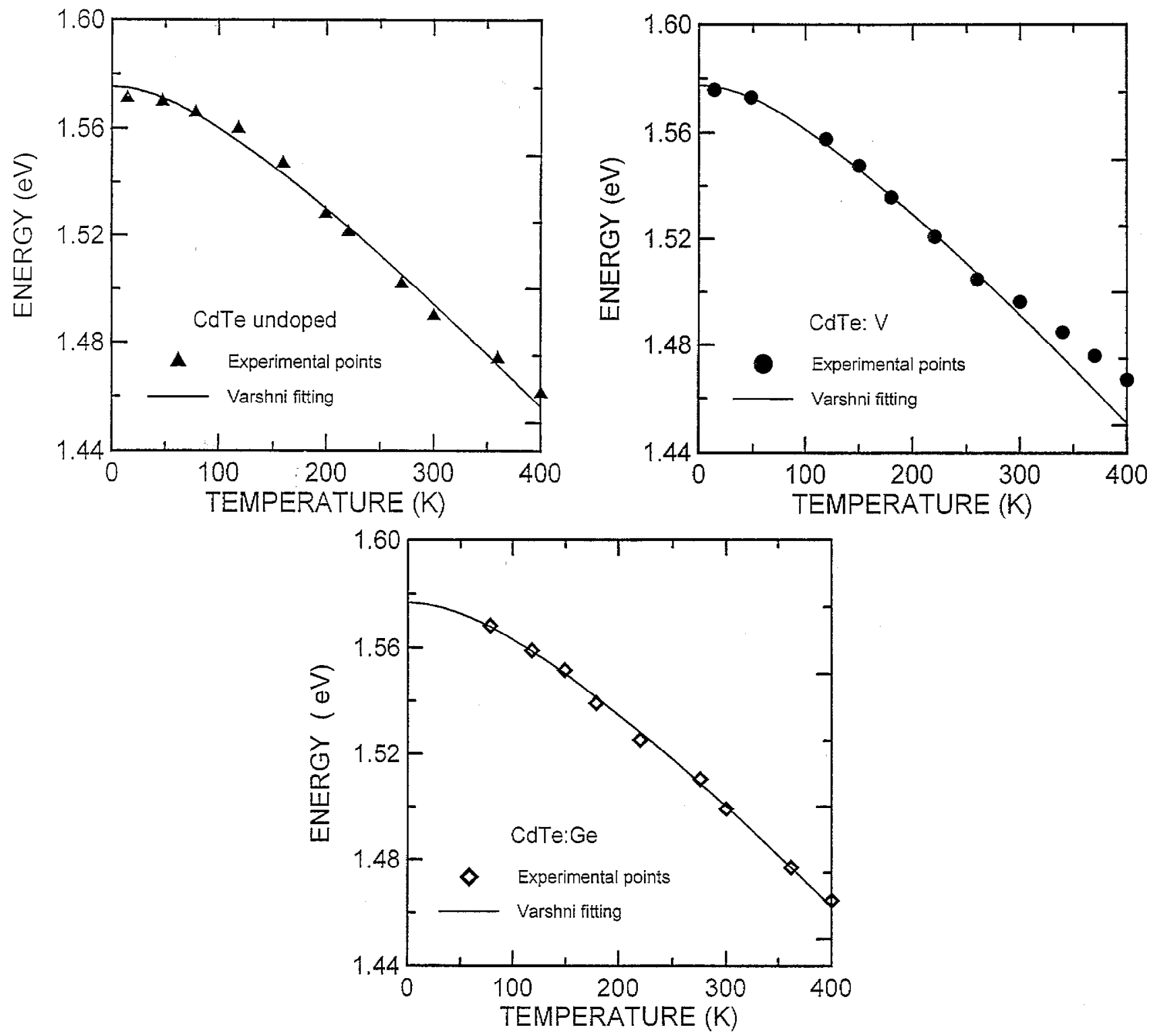

Fig. 2. Variation of $E_{0}$ with temperature for (a) undoped, (b) vanadium doped and (c) germanium doped CdTe crystals

On exciton absorption of the heavy $\left(m_{\mathrm{j}}= \pm 3 / 2\right)$ and light $\left(m_{j}= \pm 1 / 2\right)$ hole valance band splits under strain. The mounting of crystals on the cold finger of the cryostat by silver paste is likely to result in additional strain at low temperature. However, the energy difference between the two dips observed in our samples is large (about $38 \mathrm{meV}$ ) and it is quite likely that some additional source may also be contributing to this difference. It was observed that above $140 \mathrm{~K}$, the higher energy dip smeared away. Further, with the increase of temperature, the energy difference between the two dips increased slowly, which rules out the possibility of strain contribution alone. The increase of energy difference between the two dips with the increase of temperature can be understood properly, if we assign the higher energy dip to be arising from the contribution of
FKOs. With the increase of temperature, due to increase of oscillation period of FKOs, increase of energy difference between the two dips is expected. At higher temperatures, as there were no contribution from exciton or FKOs, our theoretical lineshape fitted well with the experimental lineshape and the evaluated $E_{0}$ values were in good agreement with the reported values.

Table 1

The values of Varshni parameters $\left(\alpha\right.$ and $\beta$ ) and $E_{0}$ for undoped, vanadium doped and germanium doped CdTe crystals

\begin{tabular}{llll}
\hline Sample & $E_{0}(0)(\mathrm{eV})$ & $\alpha \times 10^{4}(\mathrm{eV} / \mathrm{K})$ & $\beta(\mathrm{K})$ \\
\hline CdTe & 1.575 & 4.357 & 183.4 \\
CdTe:V & 1.578 & 4.635 & 184.5 \\
CdTe:Ge & 1.577 & 4.508 & 230.5 \\
\hline
\end{tabular}




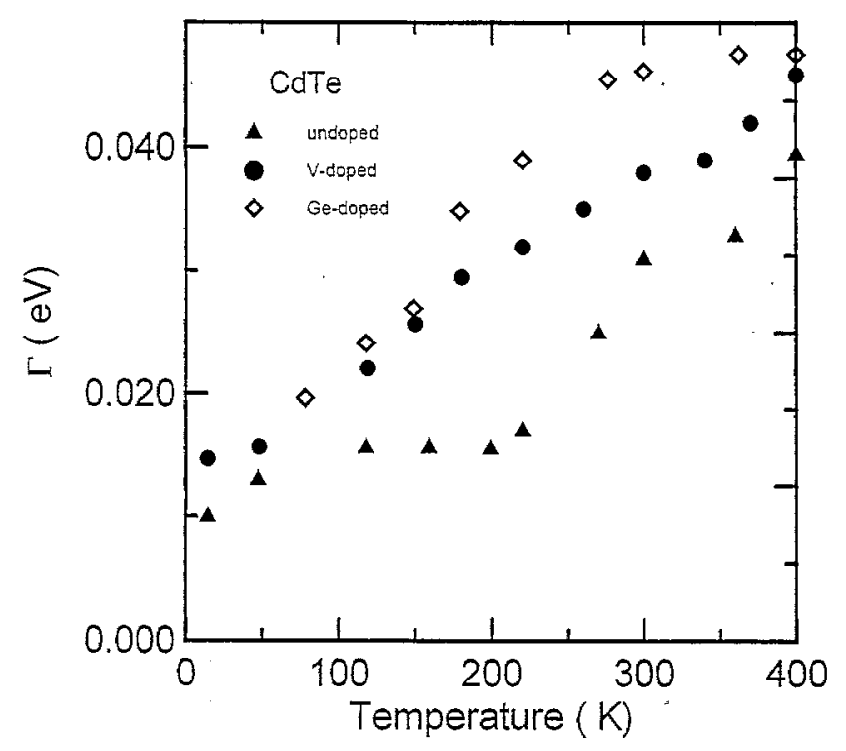

Fig. 3. Variation of energy broadening parameter $\Gamma$ with temperature for the undoped, vanadium doped and germanium doped $\mathrm{CdTe}$ crystals.

Fig. 2(a-c) represents the temperature variation of near band edge transition energy (broadly referred as the band edge energy in the text) $E_{0}$ for undoped, $\mathrm{V}$-doped and Ge-doped CdTe crystals. We note here that the variation of $E_{0}$ with temperature follows the Varshni relation [14] for all the samples rather than a linear fit as reported by some of the workers [15]. For undoped and Ge-doped crystals, the variation is fitted by only one Varshni relation for the whole range of temperature under study. However, for vanadium doped crystals the relation is valid upto a temperature of $250 \mathrm{~K}$. Varshni parameters and the fitted $0 \mathrm{~K}$ critical point energy values for the crystals are presented in Table 1. The vales of $E_{0}$ and $\alpha$ are of same order for all the crystals, whereas, $\beta$ value increased drastically on Ge doping.

The calculated values of $E_{0}$ at low temperatures are compared with the reported values at discrete temperatures [16]. We observed that our values are less than the reported values. However, the $\mathrm{E}_{0}$ values obtained for the undoped CdTe crystals are in good agreement with the values reported by Muranevich et al. [15] for their Bridgman grown crystals estimated by optical transmittance measurements. The lower values of $E_{0}$ and the failure of the model to fit the experimental lineshape properly in the low temperature spectra can be explained by considering the effect of impurity and the presence of exciton in the samples. The fact that the theoretical fit to the $14 \mathrm{~K}$ spectrum of Fig. 1 is not as good as that seen at $360 \mathrm{~K}$ suggests that the excitonic effect is playing an important role. As the band edge peak is non-symmetric in our PR spectra, broadening effect introduces a shift in the position of the band edge
[18]. The corresponding error in the determination of $E_{0}$ is generally smaller than the width of the peak/dip. The PR spectrum should contain the structures from both finite-field and zero-field spectrum as generally observed in electro-absorption (EA) spectrum. The zero-field (unmodulated) spectrum should contain structures corresponding to the bound exciton states [19]. Since the thermal broadening is much greater than the exciton binding energy (about $17 \mathrm{meV}$ for $E_{(\mathrm{X}, \mathrm{A})}$ in CdTe) [20] in our samples, it was not possible to detect the structure for exciton. The situation becomes more clear on considering the contribution from the A-centre (doner-cation vacancy pair) at low temperatures [20]. As the A-centre in CdTe is located at about $127 \mathrm{meV}$ above the valence band [20], a lower estimated value by about the same amount correspond with the superposition of A-centre transition on the band edge transition. As the resolution of our experimental setup was a restriction and the energy broadening is high in the samples, the A-centre transition could not be resolved from the band edge transition. However, the presence of such A-centre in the samples were reported by us [21] from the CL studies.

The variations of energy broadening parameter $\Gamma$ with temperature for the three samples are shown in Fig. 3. With increasing temperature, $\Gamma$ value is seen to increase for all the samples which depicts the effect of usual thermal broadening. However, the absolute value of $\Gamma$ for vanadium and germanium doped crystals are higher than the value for undoped crystals at all temperatures and this arises due to the effect of impurity broadening. We find that the broadening effect is more pronounced in Ge-doped crystals despite the fact that

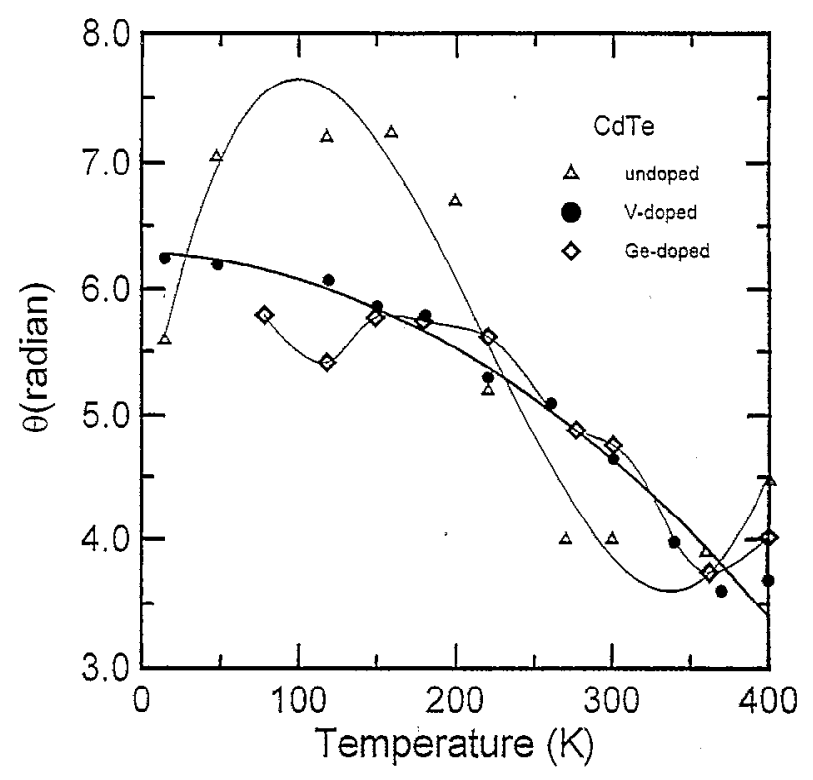

Fig. 4. Variation of phase $\theta$ with temperature for the undoped, vanadium doped and germanium doped CdTe crystals, 
the added concentration of $\mathrm{V}$ and $\mathrm{Ge}$ were of the same order. The exact cause of this effect is not yet known. A likely source could be that the cadmium vacancies $\left(V_{C d}\right)$ are being filled-up by $\mathrm{V}$ in vanadium doped crystals. The Ge plays a similar role in $\mathrm{CdTe}$, but due to higher order valence state of $\mathrm{Ge}$ ions than the $\mathrm{V}$ ions, the effect of Ge impurity is found to be more pronounced in CdTe crystals. The $\Gamma$ values evaluated for undoped crystal are of the same order as reported by other workers $[22,23]$.

With the increase of temperature, the phase factor $\theta$ is seen to decrease monotonically for the V-and Gedoped crystals (as shown in Fig. 4). It is seen that the $\theta$ value varied sinusoidally for the undoped crystals with similar trend of decrease wilh increasing temperature.

\section{Conclusions}

PR lineshape analysis is made in Bridgman grown CdTe, CdTe:V and CdTe:Ge crystals using the Aspnes theory of low field electroreflectance. Lower value of estimated $E_{0}$ at low temperatures arises due to A-centre and excitonic contribution in the band edge transition. The effects of A-centre and FKOs in the low temperature PR spectra gives rise to a bad fit of theoretical lineshape at the high energy side of the band edge transition. The $\Gamma$ value increases due to incorporation of vanadium and germanium impurities in CdTe crystals. Effect of germanium incorporation in the crystals on the increase of $\Gamma$ value is comparatively more pronounced due to its higher order valence state. From the point of view of energy broadening effect, vanadium has superiority over germanium for the preparation of semi-insulating CdTe crystals by filling the cadmium vacancy centers.

\section{Acknowledgements}

U. Pal thanks CONACyT for the financial support and the Catedra patrimonial (No. 481100-1-940460).
This work is partially supported by the CONACyT grants (Project No. 1561-E9207).

\section{References}

[1] D.E. Aspnes, in T.S. Moss (ed.), Handbook on Semiconductors, Vol. 2, North Holland, New York, 1980, p. 109.

[2] B.O. Seraphin, in R.K. Willardson and A.C. Beer (eds.), Semiconductors and Semimetals, Vol. 9, Academic, New York, 1972, p. 1 .

[3] R.N. Bhattacharya, H. Shen, P, Parayanthal, F.H. Pollak, T. Coutts and H. Aharoni, Proc. Soc. Photo-Opt. Instrum. Eng., 794 (1987) 81; Phys. Rev., B37 (1988) 4044.

[4] H. Shen, S.H. Pan, P.H. Pollak, M. Dutta and T.R. Aucoin, Phys. Rev., B36 (1987) 9384.

[5] O.J. Glembocki, B.V. Shanabrook, N. Bottka, W.T. Beard and J. Coman, Appl. Phys, Lett., 45 (1985) 970

[6] J.L. Shay, Phys. Rev., B2 (1970) 803.

[7] R.B. Bylsma, P.M. Bridenbaugh, D.H. Olson and A.M. Glass, Appl. Phys. Lett., 51 (1987) 889.

[8] E. Rzepka, Y. Marfaing, M. Cuniot and R. Triboulet, Mater. Sci. Eng., B16 (1993) 262.

[9] A. Partovi, J. Millerd, E.M. Garmire, M. Ziari, W.H. Steier, S.B. Trivedi and M.B. Klein, Appl. Phys. Lett., 57 (1990) 846.

[10] P. Moravec, M. Hage-Ali, L. Chibani and P. Siffert, Mater. Sci. Eng., B16 (1993) 223.

[11] D. Aspnes, Suff. Sci., 37 (1973) 418

[12] F.H. Pollak, in D.E. Aspnes, S. So and R.F. Potter (eds.) Optical Characterization for Semiconductor Technology, Society of Photo-Optical Instrumentation Engineers, Bellingham, WA, 1981; Proc. Soc. Photo. Opt. Inst. Eng., 276 (1981) 142.

[13] M. Sydor, J. Angelo, J.J. Wilson, W.C. Mitchel and M.Y. Yen, Phys. Rev., B40 (1989) 8473.

[14] Y.P. Varshni, Physica (Utrecht), 34 (1967) 149.

[15] A. Muranevich, M. Roitberg and E. Finkman, J. Cryst. Growth, 64 (1983) 285.

[16] D.T.F. Marple, Phys. Rev., 150 (1966) 728.

[18] B.O. Seraphin and N. Bottka, Phys. Rev., 145 (1966) 828.

[19] D.F. Blossey, Phys. Rev., B3 (1971) 1382.

[20] W. Stadler, D.M. Hofmann, H.C. Alt, T. Muschik, B.K. Meyer, E. Weigel, G. Muller-Vogt, M. Slak, E. Rupp and K.W. Benz, Phys. Rev., B51 (1995) 10619.

[21] U. Pal, J. Piqueras, P. Fernandez, M.D. Serrano and E. Dieguez, J. Appl. Phys. 76 (1994) 3720.

[22] W.S. Enloe, J.C. Parker, J. Vespoli, T.H. Myers, R.L. Harper and J.F. Schetzina, J. Appl. Phys., 61 (1987) 2005.

[23] Jaesun Lee and N.C. Giles, J. Appl. Phys., 78 (1995) 1191. 